\title{
Correlation of APOBEC3G expression with liver function indexes of patients with chronic hepatitis B and comparison in chronic hepatitis $B$, liver cirrhosis and liver cancer
}

\author{
LINA NI $^{1}$, CHUANBAO $\mathrm{LI}^{2}$ and YINGBO LI ${ }^{1}$ \\ Departments of ${ }^{1}$ Blood Transfusion and ${ }^{2}$ Hepatobiliary Surgery, Weihai Central Hospital, \\ Wendeng, Weihai, Shandong 264400, P.R. China
}

Received October 9, 2019; Accepted December 5, 2019

DOI: $10.3892 / \mathrm{ol} .2020 .11257$

\begin{abstract}
Correlation of APOBEC3G expression with liver function indexes of patients with chronic hepatitis $B$ and its expression in chronic hepatitis B, liver cirrhosis and liver cancer were investigated to evaluated the significance of APOBEC3G. Fifty-eight patients with chronic hepatitis B were selected, including 20 cases of chronic hepatitis B, 19 cases of liver cirrhosis and 19 cases of liver cancer. Liver function indexes were detected and analyzed, and messenger ribonucleic acid (mRNA) and protein expression levels of APOBEC3G in liver tissues were detected via reverse transcription-polymerase chain reaction (RT-PCR), western blotting and immunohistochemistry, followed by correlation analysis. Certain liver function indexes had significant differences among the three groups of patients $(\mathrm{P}<0.05)$. Results of RT-PCR, Western blotting and immunohistochemistry confirmed that the content of APOBEC $3 \mathrm{G}$ in liver tissues was the highest in patients with chronic hepatitis B, slightly lower in patients with liver cirrhosis and the lowest in patients with liver cancer. The content of APOBEC3G mRNA in liver tissues had a certain correlation with the content of alanine aminotransferase (ALT) $\left(r^{2}=0.34, \mathrm{P}<0.05\right)$. Other liver function indexes had no significant correlations with APOBEC3G $(\mathrm{P}>0.05)$. APOBEC $3 \mathrm{G}$ expression has a certain correlation with some liver function indexes of patients with chronic hepatitis B. There are significant differences in the expression level of APOBEC3G in patients with hepatitis, liver cirrhosis and liver cancer.
\end{abstract}

Correspondence to: Dr Yingbo Li, Department of Blood Transfusion, Weihai Central Hospital, West 3, Mishan East Road, Wendeng, Weihai, Shandong 264400, P.R. China

E-mail: zhuh3588@126.com

Key words: APOBEC3G, chronic hepatitis B, liver cirrhosis, liver cancer

\section{Introduction}

Hepatitis B virus (HBV) is one of the common infectious diseases, and the liver is the most important organ infected with HBV. Currently, there are approximately 240 million infected people and carriers of HBV in the world, and about 620,000 deaths due to HBV-related liver cirrhosis or hepatocellular carcinoma (HCC) every year (1).

APOBEC 3 is a component of innate immunity playing an important role in resisting viral invasion, including HBV and human immunodeficiency virus (HIV). In the human body, APOBEC 3 contains 7 kinds of proteins, namely APOBEC-3A, -3B, -3C, -3DE, -3F, -3G and -3H (2). There is one or two catalytic structural domains with the cytosine deaminase activity in APOBEC3, which can convert cytosine into uracil in deoxyribonucleic acid (DNA) $(3,4)$. APOBEC3, and especially APOBEC $3 \mathrm{G}$, inhibit HBV through the hyperdegeneration-dependent and hyper-degeneration-independent mechanisms $(5,6)$. APOBEC $3 \mathrm{G}$ is expressed widely in human tissues, and its messenger ribonucleic acid (mRNA) level has a close correlation with the content of lymphocytes (7). As one of the most active deaminases, APOBEC $3 \mathrm{G}$ is able to strongly inhibit the replication and editing of HBV DNA in the body (8-12).

APOBEC 3G has extremely low content in normal liver tissues, but it can exert an effect of resisting HBV infection in the body. In this study, the correlation between APOBEC3G expression level and liver function was investigated, and the influence and antiviral function on the liver was evaluated, followed by further analysis.

\section{Patients and methods}

Sample data. A total of 58 patients carrying HBV admitted in Weihai Central Hospital were selected, including 34 males and 24 females aged 45-65 years. After admission, the blood biochemical and blood routine examinations were performed, and other hepatitis virus infections were excluded. There were 20 cases clinically diagnosed with chronic hepatitis B, 19 cases with liver cirrhosis and 19 cases with liver cancer. Partial liver tissues were obtained from all patients for subsequent 
Table I. Specific data of patients.

\begin{tabular}{lccccc}
\hline Group & $\mathrm{n}$ & Male/Female & $\begin{array}{c}\text { Minimum age } \\
\text { (years) }\end{array}$ & $\begin{array}{c}\text { Maximum age } \\
\text { (years) }\end{array}$ & $\begin{array}{c}\text { Average age } \\
\text { (years) }\end{array}$ \\
\hline Chronic hepatitis B & 20 & $11 / 9$ & 45 & 63 & 60 \\
Liver cirrhosis & 19 & $11 / 8$ & 47 & 65 & 57 \\
Liver cancer & 19 & $12 / 7$ & 47 & 62 & 57 \\
\hline
\end{tabular}

Table II. Comparisons of liver function indexes among groups.

Group

\begin{tabular}{lccc}
\cline { 2 - 4 } Item & Chronic hepatitis B group & Liver cirrhosis group & Liver cancer group \\
\hline ALT $(\mathrm{U} / \mathrm{l})$ & $59.8 \pm 7.3$ & $73.5 \pm 10.2$ & $88.6 \pm 9.2^{\mathrm{a}}$ \\
AST $(\mathrm{U} / \mathrm{l})$ & $35.6 \pm 8.8$ & $47.5 \pm 9.3^{\mathrm{a}}$ & $73.7 \pm 12.1^{\mathrm{a}, \mathrm{b}}$ \\
ALP $(\mathrm{U} / \mathrm{l})$ & $87.3 \pm 16.4$ & $125.6 \pm 21.3$ & $128.7 \pm 19.6^{\mathrm{a}}$ \\
GGT $(\mathrm{U} / \mathrm{l})$ & $24.8 \pm 4.43$ & $35.7 \pm 6.5^{\mathrm{a}}$ & $48.9 \pm 7.4^{\mathrm{a}}$ \\
TP $(\mathrm{g} / \mathrm{l})$ & $65.1 \pm 13.7$ & $62.5 \pm 12.6$ & $53.1 \pm 14.7^{\mathrm{a}, \mathrm{b}}$ \\
ALB $(\mathrm{g} / \mathrm{l})$ & $42.8 \pm 10.4$ & $35.6 \pm 8.9$ & $32.1 \pm 8.5$ \\
T-BIL $(\mu \mathrm{mol} / \mathrm{l})$ & $23.3 \pm 6.5$ & $28.9 \pm 9.1$ & $29.2 \pm 4.3$ \\
D-NIL $(\mu \mathrm{mol} / \mathrm{l})$ & $4.5 \pm 1.2$ & $5.8 \pm 1.4$ & $6.1 \pm 2.3$ \\
\hline
\end{tabular}

${ }^{a}$ There is a significant difference compared with that in chronic hepatitis B group $(\mathrm{P}<0.05)$, and ${ }^{\mathrm{b}}$ there is a significant difference compared with that in liver cirrhosis group $(\mathrm{P}<0.05)$. ALT, alanine aminotransferase; AST, aspartate aminotransferase; ALP, alkaline phosphatase; GGT, $\gamma$-glutamyl transpeptidase; TP, total protein.

studies. The data of patients are shown in Table I, and there were no significant differences among groups. The study was approved by the Ethics Committee of Weihai Central Hospital and informed consents were signed by the patients and/or guardians.

RNA extraction and real-time quantitative polymerase chain reaction $(q P C R)$. RNA was extracted from the peripheral blood mononuclear cells (PBMCs) in the blood or liver tissues, and then reversely transcribed into cDNA. APOBEC3G expression level was detected on a real-time qPCR instrument (Applied Biosystems). Expression of APOBEC3G was quantified with $\beta$-actin as an internal control. Expression levels of APOBEC $3 G$ and $\beta$-actin were detected using the following primers: APOBEC $3 \mathrm{G}$ : 5'-CGGAATTCAAGCCTCACTTCAGAAACAC-3' and 5'-CGAAGCTTTCTGCCTTCCTTAGAGACTG-3', $\beta$-actin: 5'-GGACTTCGAGCAAGAGATGG-3' and 5'-AGCACT GTGTTGGCGTACAG-3'. The expression level was calculated using $2^{-\Delta \Delta \mathrm{Ct}}$ method.

Western blotting. The protein was extracted from PBMCs in the blood or liver tissues (Beyotime), and the protein concentration was detected using the protein assay kit (Shanghai Sangon). The protein sample obtained was used for western blotting. Anti-APOBEC3G monoclonal primary antibody (Cell Signaling Technology) was used in this study, and $\beta$-actin was the internal control.
Immunohistochemical staining. The rabbit anti-human APOBEC3G polyclonal antibody was purchased from Abcam. Experiments were performed strictly according to instructions of the kit (Beyotime). The color development reaction was examined under an optical microscope.

Statistical analysis. Statistical Product and Service Solutions (SPSS) 10.0 software was used for statistical processing. The correlations among variables were analyzed using Pearson's correlation coefficient $(r)$. Data are presented as mean \pm standard deviation (SD). $\mathrm{P}<0.05$ was considered to indicate a statistically significant difference.

\section{Results}

Comparisons of liver function indexes among groups. Liver function indexes in the serum in 58 patients in hepatitis B group, liver cirrhosis group and liver cancer group were detected. Results revealed that alanine aminotransferase (ALT), aspartate aminotransferase (AST), alkaline phosphatase (ALP), $\gamma$-glutamyl transpeptidase (GGT) and total protein (TP) had significant differences between hepatitis B group and liver cancer group $(\mathrm{P}<0.05)$. AST and GGT had significant differences between hepatitis B group and liver cirrhosis group $(\mathrm{P}<0.05)$. There were also significant differences in AST and TP between liver cirrhosis group and liver cancer group $(\mathrm{P}<0.05)$. The remaining indexes were not significantly different among the three groups (Table II). 


\section{A Hepatitis Liver cirrhosis Liver cancer}
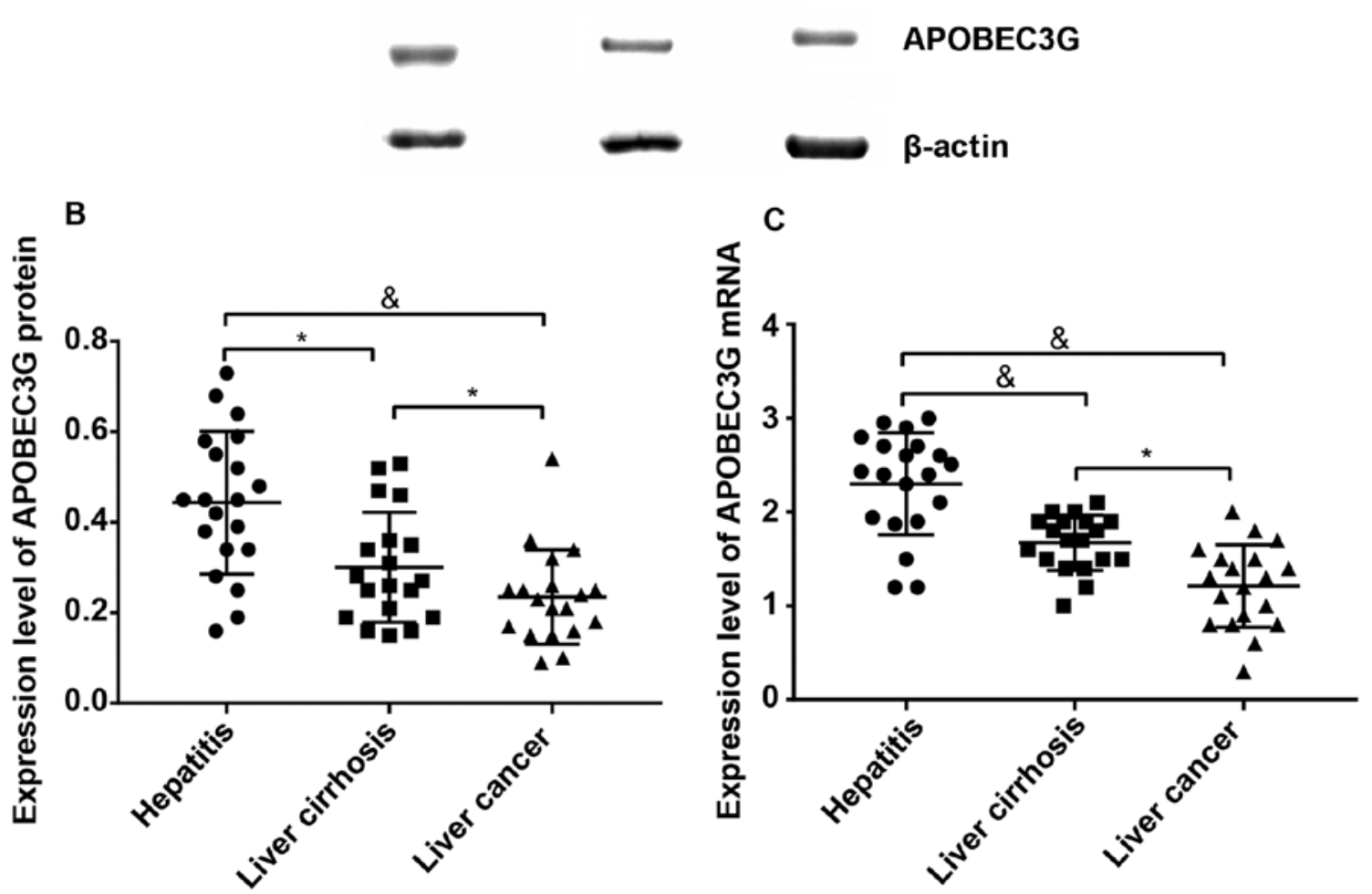

Figure 1. Comparison of APOBEC3G expression in liver tissues in patients with chronic hepatitis B, liver cirrhosis or liver cancer. (A) Detection of APOBEC3G expression in liver tissues via western blotting. (B) APOBEC3G protein expression in patients with chronic hepatitis B, liver cirrhosis and liver cancer. (C) APOBEC3G mRNA expression in patients with chronic hepatitis $\mathrm{B}$, liver cirrhosis and liver cancer $\left({ }^{*} \mathrm{P}<0.05,{ }^{\circledR} \mathrm{P}<0.01\right)$. mRNA, messenger ribonucleic acid

Detection of APOBEC3G expression in liver tissues via Western blotting and RT-PCR. The expression level of APOBEC $3 G$ protein in liver tissues was $(0.44 \pm 0.23)$ in patients with hepatitis $\mathrm{B},(0.30 \pm 0.17)$ in patients with liver cirrhosis and $(0.20 \pm 0.12)$ in patients with liver cancer, respectively, displaying significant differences among groups $(\mathrm{P}<0.05)$ (Fig. 1A and B). The expression levels of APOBEC3G mRNA in the three groups were $2.32 \pm 0.46,1.65 \pm 0.51$ and $1.21 \pm 0.37$, respectively, also showing significant differences among groups $(\mathrm{P}<0.05)($ Fig. 1C).

Immunohistochemical detection of APOBEC $3 G$ expression in liver tissues of each group. APOBEC $3 \mathrm{G}$ was expressed in liver tissues in all the three groups. The highest expression of APOBEC 3G accounted for approximately $50 \%$ in liver tissues of patients with chronic hepatitis B and only $15 \%$ in liver cancer group, and there was a significant difference between the two groups $(\mathrm{P}<0.05)$ (Table III and Fig. 2).

Correlation between APOBEC $3 G$ expression and ALT in patients with chronic hepatitis $B$. The ALT level was higher than normal in all patients, and there was a moderate correlation between APOBEC $3 \mathrm{G}$ mRNA expression level and ALT content in liver tissues $\left(r^{2}=0.34, \mathrm{P}<0.05\right)$ (Fig. 3A). There was no remarkable correlation between APOBEC $3 \mathrm{G}$ protein expression and ALT content in liver tissues $(\mathrm{P}>0.05)$ (Fig. 3B). No significant correlation was found between other liver function indexes and APOBEC $3 \mathrm{G}$ expression level $(\mathrm{P}>0.05)$.

\section{Discussion}

HBV mainly infects liver cells, and its proliferation and replication in cells will cause damage to liver cells and lead to persistent hyperplasia of liver cells, resulting in fibrosis and even carcinogenesis. APOBEC $3 \mathrm{G}$, as a component of innate immunity in the human body, has an antiviral effect, which can inhibit HBV proliferation and growth in vivo and in vitro (13-15). Virus removal and pathogenesis of $\mathrm{HBV}$ infection have close correlations with the unique interaction between virus and infected host $(16,17)$. According to studies, the risk of liver cirrhosis and HCC is extremely high for patients with a higher degree of viral replication (18).

Recently, studies have tried to clarify the influences of APOBEC 3 on HBV replication, core particle association and HBV DNA editing in vitro $(9,11,19)$. Cytidine deaminase in the production of protein diversity and immunity can remove the pathogenic and non-pathogenic exogenous DNA (20). According to the study of Stenglein et al, APOBEC3, a member of the cytidine deaminase family, can restrict the exogenous DNA in human cells (20). APOBEC3G is a member of the APOBEC 3 family, which, as a component of innate immune response, can inhibit the proliferation of DNA viruses, such as HBV $(21,22)$. Moreover, some studies have demonstrated that APOBEC3G induces C-T hyper-mutation in the newlysynthesized HBV genomic chain, resulting in the removal of hepatitis B e-antigen and decline in HBV DNA. The mode of action of APOBEC $3 \mathrm{G}$ appears to be inclusion into HBV particles through direct binding to the hepatitis B core 
A

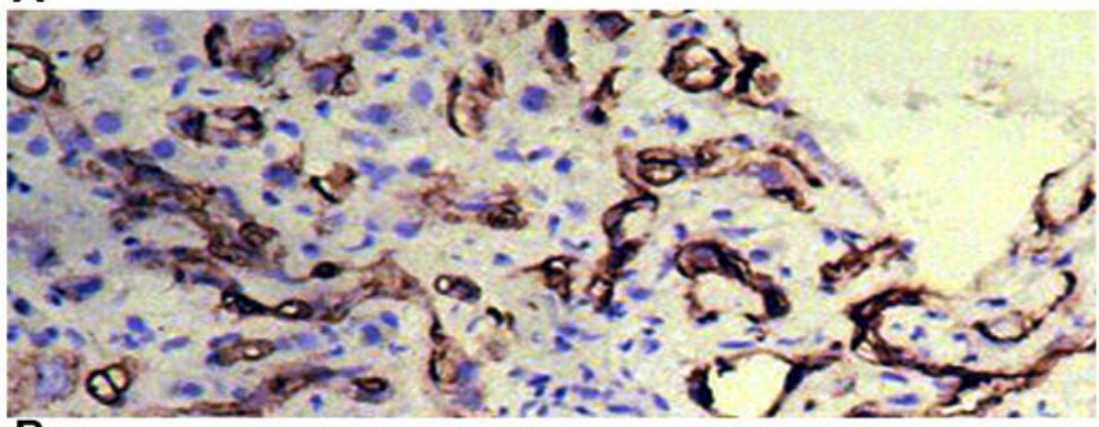

B

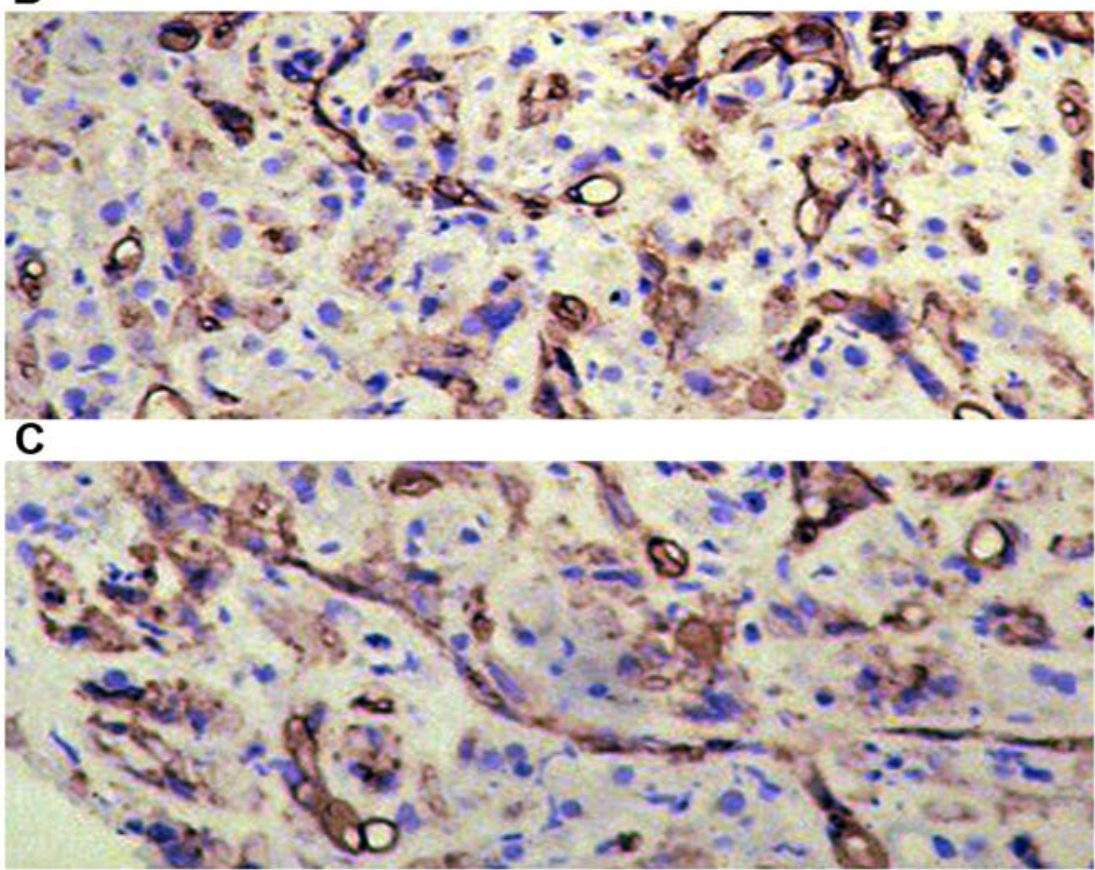

Figure 2. Immunohistochemical detection of APOBEC3G expression in liver tissues. (A) hepatitis, (B) liver cirrhosis, (C) liver cancer.

A

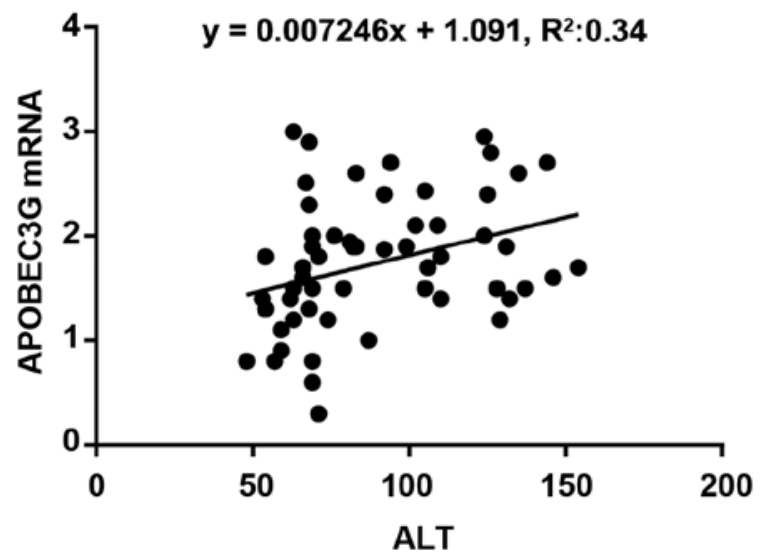

B

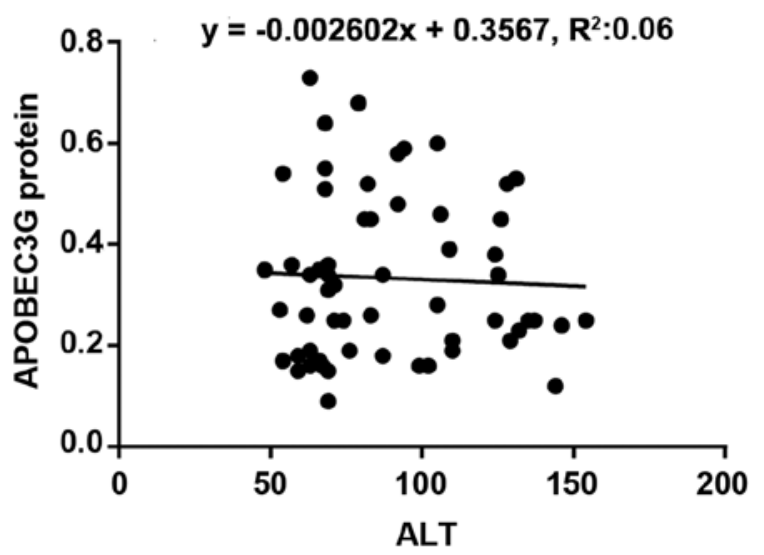

Figure 3. Correlation between APOBEC3G and ALT in patients with chronic hepatitis B. (A) Correlation between APOBEC3G mRNA and ALT in liver tissues. (B) Correlation between APOBEC $3 \mathrm{G}$ protein and ALT in liver tissues. ALT, alanine aminotransferase; mRNA, messenger ribonucleic acid.

protein (23). In addition, there are reports that interferon (IFN) can act on retrovirus and HBV non-specifically and effectively induce the production of APOBEC3G18-21 in lymphocytes and hepatocytes, indicating that IFN- $\alpha$ and IFN- $\gamma$ increase the transcription of APOBEC3G mRNA in human liver cell lines and induce high variation of HBV genome (24).

The detection of liver function indexes of patients in the three groups revealed that there were significant differences 
Table III. Immunohistochemical detection of APOBEC3G expression in liver tissues.

Expression intensity/ratio

\begin{tabular}{lccc}
\cline { 2 - 4 } Group & Positive $(+) /(\%)$ & Positive $(++) /(\%)$ & Positive $(+++) /(\%)$ \\
\hline Chronic hepatitis B group & $4 /(20.0 \%)$ & $6 /(30.0 \%)$ & $10 /(50 \%)$ \\
Liver cirrhosis group & $7 /(37 \%)$ & $5 /(26 \%)$ & $7 /(37 \%)$ \\
Liver cancer group & $12 /(63 \%)^{\mathrm{a}}$ & $4 /(21 \%)$ & $3 /(15 \%)^{\mathrm{a}}$
\end{tabular}

${ }^{a}$ There is a significant difference compared with that in chronic hepatitis B group $(\mathrm{P}<0.05)$.

in some liver function indexes between any two groups, but no obvious rules were found in indexes with significant differences between two groups in our analysis.

Considering the interaction between APOBEC $3 \mathrm{G}$ and HBV, in this study, albumin was selected as an index reflecting the protein anabolic function of hepatocytes, ALT and AST were selected as indexes reflecting whether there was damage to hepatocytes and its severity, and total bilirubin was selected as an index displaying liver-gallbladder excretion, secretion and detoxification, and the correlation with APOBEC3G in liver tissues was analyzed, so as to investigate the correlation between liver function and APOBEC3G in patients with chronic hepatitis B. Results manifested that the APOBEC3G mRNA level had a certain correlation with ALT content in liver tissues $\left(r^{2}=0.34, \mathrm{P}<0.05\right)$, but other liver function indexes involved in this study had no remarkable correlations with APOBEC3G $(\mathrm{P}>0.05)$. According to results of this study, some liver function indexes had a certain correlation with APOBEC 3G, but most indexes had no correlation. APOBEC 3G, as a component of innate immune response, can inhibit HBV proliferation without direct influence on the liver function, but the interaction between APOBEC3G and liver function remains to be further studied.

APOBEC $3 \mathrm{G}$ content in patients with chronic hepatitis B, liver cirrhosis and liver cancer was analyzed in this study. Results revealed that the content of APOBEC $3 \mathrm{G}$ in liver tissues was the highest in patients with chronic hepatitis $B$, slightly lower in patients with liver cirrhosis and the lowest in patients with liver cancer, indicating that the expression level of APOBEC3G may partially display the damage degree of the liver. It was further confirmed via immunohistochemistry that APOBEC3G was expressed in liver tissues in all the three groups. The expression intensity of APOBEC $3 \mathrm{G}$ was the strongest in liver tissues of patients in chronic hepatitis B group, accounting for the highest proportion, while it was slightly weaker in liver cirrhosis group and the weakest in liver cancer group, accounting for the lowest proportion.

This study suggests that APOBEC $3 \mathrm{G}$ expression has a certain correlation with some of the liver function indexes of patients with chronic hepatitis B. There are significant differences in the expression level of APOBEC $3 \mathrm{G}$ in patients with hepatitis, liver cirrhosis and liver cancer. Larger sample size is required for a confirmational study to evaluate the correlation of APOBEC3G with liver function indexes of patients with chronic hepatitis $\mathrm{B}$.

\section{Acknowledgements}

Not applicable.

\section{Funding}

No funding was received.

\section{Availability of data and material}

The datasets used and/or analyzed during the current study are available from the corresponding author on reasonable request.

\section{Authors' contributions}

LN drafted the mansucript. LN and CL collected and analyzed the general information of patients. LN and YL worked on qPCR and Western blot analysis. All authors read and approved the final manuscript.

\section{Ethics approval and consent to participate}

The study was approved by the Ethics Committee of Weihai Central Hospital and informed consents were signed by the patients and/or guardians.

\section{Patient consent for publication}

Not applicable.

\section{Competing interests}

The authors declare that they have no competing interests

\section{References}

1. Gerlich WH: Medical virology of hepatitis B: How it began and where we are now. Virol J 10: 239, 2013.

2. Teng B, Burant CF and Davidson NO: Molecular cloning of an apolipoprotein B messenger RNA editing protein. Science 260: 1816-1819, 1993

3. Wedekind JE, Dance GS, Sowden MP and Smith HC: Messenger RNA editing in mammals: New members of the APOBEC family seeking roles in the family business. Trends Genet 19: 207-216, 2003.

4. Deng Y, Du Y, Zhang Q, Han X and Cao G: Human cytidine deaminases facilitate hepatitis B virus evolution and link inflammation and hepatocellular carcinoma. Cancer Lett 343: 161-171, 2014. 
5. Nguyen DH, Gummuluru S and Hu J: Deamination-independent inhibition of hepatitis B virus reverse transcription by APOBEC3G. J Virol 81: 4465-4472, 2007.

6. Rösler C, Köck J, Kann M, Malim MH, Blum HE, Baumert TF and von Weizsäcker F: APOBEC-mediated interference with hepadnavirus production. Hepatology 42: 301-309, 2005.

7. Koning FA, Newman EN, Kim EY, Kunstman KJ, Wolinsky SM and Malim MH: Defining APOBEC3 expression patterns in human tissues and hematopoietic cell subsets. J Virol 83: 9474-9485, 2009.

8. Vartanian JP, Henry M, Marchio A, Suspène R, Aynaud MM, Guétard D, Cervantes-Gonzalez M, Battiston C, Mazzaferro V, Pineau P, et al: Massive APOBEC3 editing of hepatitis B viral DNA in cirrhosis. PLoS Pathog 6: e1000928, 2010.

9. Turelli P, Mangeat B, Jost S, Vianin S and Trono D: Inhibition of hepatitis B virus replication by APOBEC3G. Science 303: 1829, 2004.

10. Köck $\mathrm{J}$ and Blum HE: Hypermutation of hepatitis $\mathrm{B}$ virus genomes by APOBEC 3G, APOBEC3C and APOBEC3H. J Gen Virol 89: 1184-1191, 2008.

11. Henry M, Guétard D, Suspène R, Rusniok C, Wain-Hobson S and Vartanian JP: Genetic editing of HBV DNA by monodomain human APOBEC3 cytidine deaminases and the recombinant nature of APOBEC3G. PLoS One 4: e4277, 2009.

12. Suspène R, Guétard $D$, Henry $M$, Sommer $P$, Wain-Hobson $S$ and Vartanian JP: Extensive editing of both hepatitis B virus DNA strands by APOBEC 3 cytidine deaminases in vitro and in vivo. Proc Natl Acad Sci USA 102: 8321-8326, 2005.

13. Soros VB and Greene WC: APOBEC3G and HIV-1: Strike and counterstrike. Curr Infect Dis Rep 8: 317-323, 2006.

14. Chiu YL and Greene WC: Multifaceted antiviral actions of APOBEC3 cytidine deaminases. Trends Immunol 27: 291-297, 2006.

15. Svarovskaia ES, Xu H, Mbisa JL, Barr R, Gorelick RJ, Ono A, Freed EO, Hu WS and Pathak VK: Human apolipoprotein B mRNA-editing enzyme-catalytic polypeptide-like 3G (APOBEC3G) is incorporated into HIV-1 virions through interactions with viral and nonviral RNAs. J Biol Chem 279: 35822-35828, 2004.
16. Bonvin M, Achermann F, Greeve I, Stroka D, Keogh A, Inderbitzin D, Candinas D, Sommer P, Wain-Hobson S, Vartanian JP, et al: Interferon-inducible expression of APOBEC3 editing enzymes in human hepatocytes and inhibition of hepatitis B virus replication. Hepatology 43: 1364-1374, 2006.

17. El-Zayadi AR: Hepatitis B virus infection: The Egyptian situation. Arab J Gastroenterol 8: 94-98, 2007.

18. Guan R, Yap I, Wong L, Tan LH, Oon CJ and Wee A: Evidence of viral replication in HBsAg positive patients with hepatocellular carcinoma: Measurement of serum hepatitis B virus deoxyribonucleic acid (HBV-DNA). Ann Acad Med Singapore 18: 8-11, 21989, 1989.

19. Zhao D, Wang X, Lou G, Peng G, Li J, Zhu H, Chen F, Li S, Liu D, Chen Z, et al: APOBEC3G directly binds Hepatitis B virus core protein in cell and cell free systems. Virus Res 151: 213-219, 2010.

20. Stenglein MD, Burns MB, Li M, Lengyel J and Harris RS: APOBEC3 proteins mediate the clearance of foreign DNA from human cells. Nat Struct Mol Biol 17: 222-229, 2010.

21. Vartanian JP, Henry M, Marchio A, Suspene R, Aynaud MM, Guetard D, Cervantes-Gonzalez M, Battiston C, Mazzaferro V, Pineau P et al: Massive APOBEC3 editing of hepatitis B viral DNA in cirrhosis. PLoS Pathog 6: e1000928, 2010.

22. Noguchi C, Hiraga N, Mori N, Tsuge M, Imamura M, Takahashi S, Fujimoto Y, Ochi H, Abe H, Maekawa T, et al: Dual effect of APOBEC3G on Hepatitis B virus. J Gen Virol 88: 432-440, 2007.

23. Seppen J: Unedited inhibition of HBV replication by APOBEC3G. J Hepatol 41: 1068-1069, 2004

24. Tanaka Y,MarusawaH,SenoH, Matsumoto Y,Ueda Y,Kodama Y, Endo Y, Yamauchi J, Matsumoto T, Takaori-Kondo A, et al: Anti-viral protein APOBEC3G is induced by interferon-alpha stimulation in human hepatocytes. Biochem Biophys Res Commun 341: 314-319, 2006. 